\title{
CONCIENCIA FONOLÓGICA EN NIÑOS PREESCOLARES DE 4 Y 5 AÑOS
}

\author{
PHONOLOGICAL AWARENESS IN \\ PRESCHOOL CHILDREN
}

\author{
Carmen Julia Coloma Tirapegui $^{1}$ \\ Isabel Cobarrubias Bravo \\ Zulema De Barbieri Ortiz ${ }^{1}$ \\ ${ }^{1}$ Profesor Asistente, Escuela de Fonoaudiología, Universidad de Chile, Magíster en Educación Especial, \\ Pontificia Universidad Católica de Chile. \\ ${ }^{2}$ Asistente de investigación, Escuela de Fonoaudiología, Universidad de Chile. Licenciada en \\ Fonoaudiología, Universidad de Chile.
}

Correspondencia a: ccoloma@med.uchile.cl

\begin{abstract}
RESUMEN
El objetivo de este estudio es conocer el desempeño de la conciencia fonológica en preescolares chilenos. Para ello, se trabajó con un grupo de estudio constituido por 120 niños chilenos de nivel socioeconómico bajo. La muestra se dividió en 2 grupos: 60 niños de 4 años y 60 de 5 años. Se aplicó una prueba destinada a evaluar habilidades metalingüísticas de tipo fonológico. Los resultados obtenidos indican que los preescolares de 5 años evidencian un rendimiento significativamente mayor que el grupo de niños de 4 años en tareas de conciencia fonológica. Lo anterior, permite concluir que existe un incremento de las habilidades metalingüísticas relacionadas con la sílaba al comparar preescolares de 4 y 5 años. Sin embargo, en los niños de 5 años no se advierte un conocimiento metafonológico de la sílaba totalmente establecido.
\end{abstract}

Palabras Clave: Conciencia del fonema; Conciencia fonológica; Conciencia silábica; Desarrollo lingüístico; Habilidades metalingüísticas.

\begin{abstract}
The aim of this study is to know the performance phonological awareness in preschool Chilean children. A metaphonological awareness test is applied to 60 children of four years old and 60 children of five years old who come from low socioeconomic income families. The results show that the preschool of 5 year-old a significantly major yield that the group of 4-year-old children in tasks of phonological awareness. So, the results indicate an increase in children's syllable phonological awareness development according to chronological age. However, no evidence states a complete acquisition of syllable's metaphonological knowledge at five years old.
\end{abstract}

Key Words: Phoneme awareness; Phonological awareness; Syllable awareness; Linguistic development; Metalinguistics abilities. 


\section{INTRODUCCIÓN}

La conciencia fonológica es una habilidad metalingüistica que permite identificar y manipular tanto las sílabas como los fonemas que constituyen una palabra1. Según las unidades fonológicas previamente mencionadas se distinguen dos tipos: la conciencia de la sílaba y la conciencia del fonema.

La conciencia silábica es el conocimiento explícito de que las palabras están constituidas por sílabas. Por su parte, la conciencia del fonema implica la habilidad para manipular los fonemas que componen un término2. De este modo, existe acuerdo con respecto a las unidades que están involucradas en la conciencia fonológica.

Otro aspecto que se ha considerado parte de esta habilidad metafonológica es la rima, no obstante, es un tema controvertido, dado que existen dos posturas al respecto. Una de ellas plantea que la identificación de la rima sólo implica un proceso perceptivo y por ello no es posible entenderla como una habilidad metafonológica. Por el contrario, la otra perspectiva afirma que esta unidad es una manifestación de la conciencia fonológica, ya que su identificación requiere abstraer los segmentos fonológicos finales de las palabras que se comparan ${ }^{3}$.

La conciencia del fonema cumple un rol fundamental en el aprendizaje de la decodificación lectora porque permite comprender que los fonemas están representados en grafemas y la manera en que esta representación se manifiesta en las palabras. También, contribuye a utilizar la correspondencia fonema-grafema para decodificar los términos impresos en el texto ${ }^{4,5}$. Además, se ha demostrado que existe una fuerte relación recíproca entre esta habilidad metafonológica y el aprendizaje de la lectura. Una de las consecuencias de esta asociación es que los niños con dificultades en el aprendizaje lector evidencian problemas en la conciencia fonológica6.

El desarrollo de la conciencia fonológica no está totalmente consensuado. Sin embargo, se sabe que la adquisición de la conciencia silábica es anterior a la del fonema. 
Así, la habilidad metalingüística de la sílaba se manifiesta en la etapa preescolar y no requiere de la decodificación para su desarrollo, en consecuencia, es independiente del proceso de alfabetización ${ }^{8}$. Al respecto, se ha observado que niños en la etapa preescolar han adquirido la mayoría de las habilidades relacionadas con la sílaba y no las correspondientes a la conciencia del fonema9.

Por su parte, la habilidad metafonológica del fonema aparece en el periodo escolar y su desarrollo está determinado por el aprendizaje de la decodificación ${ }^{10}$. De hecho, se ha advertido que los niños que aprenden a leer mediante el método fónico incrementan las habilidades metalingüísticas del fonema en la medida en que aumentan su lectura ${ }^{11}$. Lo anterior, sugiere que el proceso de alfabetización es central para que se adquiera este tipo de conciencia.

En síntesis, los niños en la etapa preescolar desarrollan las habilidades metafonológicas relacionadas con la sílaba y en el periodo escolar adquieren la conciencia del fonema.

Aún cuando es aceptada esta secuencia evolutiva, la evidencia sobre las características que presenta la conciencia de la sílaba en preescolares hablantes del español es limitada. Por ello, el objetivo de este trabajo es conocer el desempeño de la conciencia fonológica en preescolares chilenos de cuatro y cinco años.

\section{MÉTODO}

Para la realización de este estudio se seleccionaron 120 preescolares chilenos sin problemas de lenguaje de nivel socioeconómico bajo. Por ello, se excluyeron los menores que estaban en tratamiento fonoaudiológico o que presentaban antecedentes de problemas de lenguaje. Estos antecedentes se obtuvieron de la ficha escolar y de la información que entregaron los padres sobre el desarrollo lingüístico de sus hijos.

Se consideró importante controlar este aspecto porque un adecuado desarrollo del lenguaje es necesario para la adquisición de la conciencia fonológica ${ }^{12}$.

El nivel socioeconómico se estableció a partir de la información obtenida de la ficha CAS-2 (instrumento destinado a estratificar socialmente a las familias que postulan a 
beneficios sociales en Chile). Es importante destacar que el género no se controló, porque existe evidencia que este factor no influye en el desarrollo de la conciencia fonológica 9,13.

Los 120 niños seleccionados asistían al jardín infantil y tenían entre cuatro y cinco años 11 meses. Considerando sus edades, se distinguieron dos grupos de estudio. El primer grupo estaba constituido por 60 niños (26 hombres y 34 mujeres) cuyo rango de edad fluctuaba entre los cuatro años y cuatro años 11 meses con un promedio de cuatro años, diez meses. El segundo grupo estaba constituido por 60 niños (27 hombres y 33 mujeres) cuyo rango de edad fluctuaba entre los cinco años y los cinco años 11 meses y un promedio de cinco años, cinco meses.

La conciencia fonológica se evaluó utilizando la "Prueba Destinada para Evaluar Habilidades Metalingüísticas de Tipo Fonológico" ${ }^{14}$. Se seleccionó este instrumento porque mide fundamentalmente habilidades metalingüísticas que se desarrollan en la etapa preescolar. Por ello, la mayoría de las subpruebas se centran en la evaluación de la conciencia silábica. Cada subprueba está constituida de ocho ítemes y cuenta con un ejemplo que se usa para la comprensión de la instrucción.

Según lo anterior, las primeras cuatro subpruebas están destinadas a la evaluación de la conciencia de la sílaba, la quinta se centra en la representación fonema-grafema y la última aborda la conciencia del fonema.

Las subpruebas que evalúan la conciencia silábica son: identificación de rima, identificación de la sílaba inicial, análisis de la palabra en sílabas e inversión de las sílabas. En las tareas de identificación el tipo predominante de sílaba es la constituida por consonante más vocal (sílaba libre), sin embargo, en algunos ítemes hay términos con sílabas de una vocal o con sílabas trabantes. En la actividad de análisis, las palabras están formadas por sílabas libres y su metría fluctúa entre una y cuatro sílabas. Finalmente en la inversión, los términos se constituyen de dos sílabas libres. 
La subprueba que mide asociación fonema-grafema considera los fonemas: /t/, /l/, /p/, $\mid \mathrm{X} /, / \mathrm{m} /, / \mathrm{k} /$, /f/, /s/ y subprueba que evalúa la síntesis fonémica utiliza palabras conformadas por dos sílabas libres.

La corrección de la prueba consiste en asignar un punto a cada respuesta correcta y cero a las incorrectas 0 a las sin contestar. El puntaje total de la prueba es 48 y cada subprueba tiene un máximo de ocho puntos.

La evaluación se realizó en forma individual en una sala del establecimiento donde solo se encontraba la evaluadora y el niño. Antes de aplicar cada subprueba la examinadora se aseguraba, mediante un ejemplo, de que el niño comprendiera las instrucciones. Todo el proceso de evaluación tuvo una duración aproximada de 20 minutos. Finalmente, es importante destacar que se solicitó a los padres o tutores de los niños el consentimiento informado para evaluarlos.

Los resultados son expuestos de manera descriptiva e incluyen la comparación de los desempeños de ambos grupos y los porcentajes de rendimiento. La comparación se efectuó utilizando la prueba no paramétrica $U$ de Mann Whitney. Por su parte, los porcentajes se obtuvieron de los promedios de la totalidad de la prueba y de los de cada subprueba en los dos rangos de edad.

\section{RESULTADOS}

La tabla 1 presenta la comparación del desempeño de ambos grupos de niños en la Prueba de Habilidades Metafonológicas.

Tabla 1

Comparación del desempeño total en la prueba de habilidades metafonológicas entre ambos grupos de niños.

\begin{tabular}{ccc}
\hline Grupo & Promedio & U Mann Whitney \\
\hline 4 años & 22.53 & 974.5 p: $0.000^{*}$ \\
\hline 5 años & 28.05 & \\
\hline${ }^{*}$ p 0.05 & \\
"Cobarrubias, l.; Hidalgo, P.; Parada, S.; Pozo, F. 2005. Conciencia Fonológica y Habilidades Cognitivas no Verbales en niños de \\
4 y 5 años. Seminario de Título para optar al grado de Licenciado en Fonoaudiología. Facultad de Medicina. Universidad de Chile.
\end{tabular}

Los resultados expuestos en la tabla 1 muestran que existe diferencia significativa entre los niños de ambos grupos de edad. Se observa que los niños de cinco años presentan 
un desempeño superior al de los de cuatro años, lo que sugiere que las habilidades metafonológicas se incrementan en este periodo.

También fue importante comparar los rendimientos que los participantes lograban en cada una de las subpruebas para establecer si el incremento en la conciencia fonológica estaba determinado por todas las habilidades o solo por algunas. La tabla 2 ilustra estos resultados.

Tabla 2

Comparación del desempeño en cada una de las subpruebas en ambos grupos de niños.

\begin{tabular}{llc}
\hline \multicolumn{1}{c}{ Subprueba } & Grupo & U Mann Whitney \\
\hline Reconocimiento de & 4 años & 997 \\
sonido final & 5 años & p: $0.000^{*}$ \\
\hline \multirow{2}{*}{ Reconocimiento de sonido inicial } & 4 años & 1086.5 \\
& 5 años & p: $0.000^{*}$ \\
\hline Identificación del número de & 4 años & 1219.5 \\
sílabas & 5 años & p: $0.000^{*}$ \\
\hline \multirow{2}{*}{ Inversión silábica } & 4 años & 1741 \\
& 5 años & p: 0.753 \\
Asociación fonema grafema & 4 años & 1194.5 \\
\hline \multirow{2}{*}{ Análisis fonémico } & 5 años & p: $0.001^{*}$ \\
\hline
\end{tabular}

$\mathrm{p} \leq 0.05$

"Cobarrubias, I.; Hidalgo, P.; Parada, S.; Pozo, F. 2005. Conciencia Fonológica y Habilidades Cognitivas no Verbales en niños de 4 y 5 años. Seminario de Título para optar al grado de Licenciado en Fonoaudiología. Facultad de Medicina. Universidad de Chile.

En la tabla 2 se observa que existen diferencias significativas en la mayoría de las subpruebas a favor de los niños de cinco años (Reconocimiento Final de Sonido, Reconocimiento de Sonido Inicial, Identificación del Número de Sílabas y Asociación FonemaGrafema). En las subpruebas Inversión Silábica y Análisis Fonémico ambos grupos de niños tienen un rendimiento similar, lo que indica que estas habilidades no aumentan en esta etapa.

Los análisis relacionados con los porcentajes de rendimiento de ambos grupos etáreos en la totalidad de la prueba y en cada una de las subpruebas se presentan en las tablas 3 y 4 . 


\section{Tabla 3}

Porcentaje de rendimiento total en la prueba de habilidades metafonológicas en ambos grupos de niños.

\begin{tabular}{ccl}
\hline Grupo & Promedio & $\%$ \\
\hline 4 años & 22.53 & 47 \\
\hline 5 años & 28.05 & 58 \\
\hline
\end{tabular}

En la tabla 3 se evidencia que los niños de cuatro años alcanzan un rendimiento bajo el $50 \%$ en toda la prueba, en cambio, los de cinco años presentan un desempeño superior a este porcentaje. Aún cuando el grupo de los niños mayores evidencian un incremento en su desempeño metalingüístico no logran un rendimiento superior al $60 \%$.

\section{Tabla 4}

Porcentaje de rendimiento en las subpruebas de habilidades metafonológicas en ambos grupos de niños.

\begin{tabular}{lccc}
\hline \multicolumn{1}{c}{ Subprueba } & Grupo & Promedio & $\%$ \\
\hline Reconocimiento de & 4 años & 3.36 & 42 \\
sonido final & 5 años & 4.93 & 62 \\
\hline Reconocimiento de sonido & 4 años & 3.1 & 39 \\
inicial & 5 años & 4.23 & 53 \\
\hline Identificación del número de & 4 años & 4.76 & 60 \\
sílabas & 5 años & 5.7 & 71 \\
\hline \multirow{2}{*}{ Inversión silábica } & 4 años & 3.7 & 46 \\
& 5 años & 3.75 & 47 \\
\hline \multirow{2}{*}{ Asociación fonema grafema } & 4 años & 3.5 & 44 \\
\hline \multirow{2}{*}{ Análisis fonémico } & 5 años & 4.83 & 60 \\
\hline
\end{tabular}

La tabla 4 muestra que en todas las subpruebas los niños de cinco años obtienen mayores porcentajes en sus rendimientos que los de cuatro años. Es destacable que obtienen desempeños iguales o superiores al $60 \%$ en tres subpruebas (Reconocimiento de Sonido Final, Identificación del Número de Sílabas y Asociación Fonema-Grafema), por el contrario, los participantes de cuatro años solo alcanzan un $60 \%$ en la subprueba Identificación del Número de Sílabas. 
En la subprueba Inversión Silábica los preescolares de cinco años presentan su menor rendimiento, mientras que los de cuatro años lo evidencian en la subprueba Reconocimiento de Sonido Inicial.

\section{DISCUSIÓN}

Los datos de la presente investigación muestran que existe un incremento en la conciencia fonológica entre los cuatro y los cinco años. Especialmente, en aquellas habilidades metalingüísticas relacionadas con las tareas de identificación y segmentación de sílabas. Aún cuando se observa desarrollo en estas habilidades el rendimiento del grupo de cinco años no supera el $60 \%$, lo que indica que la conciencia fonológica está en proceso de adquisición.

La tarea más fácil para ambos grupos fue la segmentación silábica, lo que concuerda con lo encontrado en un estudio realizado con niños españoles de cuatro años ${ }^{5}$. En la investigación citada estos menores alcanzan un $77 \%$ de rendimiento, en cambio, los participantes chilenos de la misma edad obtienen un $60 \%$. Un rendimiento semejante al observado en los niños españoles se advierte en los preescolares chilenos de cinco años. Es importante destacar que las pruebas utilizadas, si bien consideraban los mismos aspectos y modalidad de evaluación, diferían en la cantidad de estímulos presentados.

El porcentaje de rendimiento en la tarea de identificación de rima de los preescolares de cuatro años es semejante al encontrado en niños españoles de la misma edad5. En ambos grupos (chilenos y españoles) se observa que esta actividad es difícil, ya que los rendimientos logrados no superan el 50\% (chilenos: 42\% y españoles: 46\%). Otra investigación muestra que la rima no está totalmente afianzada en estas edades, en ella se concluye que esta tarea es difícil para los preescolares ${ }^{15}$.

La identificación del sonido inicial fue una de las tareas que presentó mayor dificultad para ambos grupos de niños (39\% para los niños de cuatro años y $53 \%$ para los de cinco años). Este resultado es consistente con lo encontrado uno de los trabajos previamente 
citados $^{5}$. Por el contrario, esta evidencia se opone a lo observado en otra investigación ${ }^{10}$ donde la identificación del sonido inicial fue fácil para los participantes de su estudio.

La discrepancia previamente aludida puede deberse a que los niños de este estudio ${ }^{10}$ eran mayores (promedio de edad cinco años, ocho meses) que los participantes de nuestra investigación (promedio de edad cinco años, cinco meses). En consecuencia, es posible que esta habilidad se encuentre en proceso de adquisición en los preescolares chilenos que participaron en este trabajo.

Otra tarea difícil que presentó rendimientos similares en ambos grupos fue la inversión silábica (niños de cuatro años: $46 \%$ y niños de cinco años: $47 \%$ ). Este resultado es concordante con datos que indican que la inversión silábica no progresa en niños de cinco años aunque se los entrene en esta habilidad². Lo anterior sugiere que esta manifestación de la conciencia silábica no se incrementa en las edades estudiadas.

El análisis fonémico fue otra habilidad en que no se observó aumento en ambos grupos, este resultado es factible ya que al ser una habilidad metafonológica relacionada con el fonema se espera que su desarrollo se manifieste fundamentalmente en la etapa escolar ${ }^{10}$.

Finalmente, la asociación fonema-grafema se incrementa a los cinco años, revelando que los niños aumentan su conocimiento sobre los sonidos de las letras durante este periodo. Este fenómeno no es sorprendente porque en la etapa preescolar a los niños se les enseñan los grafemas de algunos fonemas. Lo anterior fue constatado en un estudio realizado en niños entre cinco y siete años que iniciaban su etapa escolar donde se observó que conocían la totalidad de las vocales y varias de las consonantes que se utilizaron en esta subprueba ${ }^{16}$.

En síntesis, los resultados obtenidos en esta investigación permiten concluir que existe un incremento en las habilidades metalingüísticas relacionadas con la sílaba al comparar niños de cuatro y cinco años. Sin embargo, es destacable que a los cinco años no se observa un conocimiento metafonológico de la sílaba absolutamente establecido. Este resultado no concuerda con hallazgos encontrados en niños hispanohablantes de la misma edad en que se ha advertido que esta habilidad metafonológica está afianzada en este grupo 
etario². Es posible que esta discrepancia esté dada por el hecho de que los participantes de este estudio pertenecen al nivel socioeconómico bajo.

Al respecto, existe evidencia que el desarrollo lingüístico general está influido por la calidad del lenguaje oral del ambiente en el que los niños están insertos. A su vez, se sabe que el entorno lingüístico del nivel socioeconómico medio-bajo no favorece plenamente la adquisición de habilidades lingüísticas y metalingüísticas ${ }^{17}$. De hecho, en Chile se ha determinado que niños de nueve años de nivel socioeconómico medio-bajo presentan un desempeño descendido en habilidades psicolingüísticas necesarias para el aprendizaje de la lectura ${ }^{18}$.

\section{REFERENCIAS}

1. Clemente, M. \& Domínguez, A. (1999) La enseñanza de la lectura. Enfoque psicolingüístico y sociocultural, Madrid: Pirámide.

2. Carrillo, M.S. \& Marín, J. (1996) Desarrollo Metafonológico y adquisición de la lectura: un estudio de entrenamiento, Madrid: CIDE, MEC.

3. Naremore, R.; Densmore, A. \& Harman, D. (2001) Phonological Awareness. Assessment and treatment of school-age language disorders a resourse manual. Canada: Singular, Thomson Learning.

4. Torgesen, J.; Otaiba, S. \& Grek, M. (2005) Assessment and Instruction for Phonemic Awareness and word Recognition Skills. En Catts H, Kamhi A. Language and Reading Disabilities, $2^{\mathrm{a}}$ ed. Boston: Pearson; p. 127-151.

5. Herrera, L. \& Defior, S. (2005) Una aproximación al procesamiento fonológico de los niños prelectores: Conciencia Fonológica, Memoria Verbal a corto plazo y Denominación, Psykhe, Vol. 14, 81-95.

6. Cherd, D. \& Dickson, S. (1999). Phonological Awareness: Instructional and Assessment Guidelines, Learning Disabilities, Vol. 34, 261-270.

7. Morais, J., Alegría J. \& Content, A. (1987). Segmental awareness: Respectable, useful and almost always necessary. Curr Psychol Cogn., Vol. 7, 530-556.

8. Gomez-Betancur, L.A., Pineda, D.A. \& Aguirre-Acevedo, D.C. (2005) Conciencia Fonológica en niños con trastorno de la atención sin dificultades en el aprendizaje. Rev. Neurol., Vol.40, 581-586. 
9. Sales de Meneses, M., Passos Lozi, G., Regina de Souza, L., \& Asensio-Ferreira, V.J. (2004). Conciencia Fonológica: Diferenças entre meninos y meninas. Revista CEFAC, Vol. 6 , 242-246.

10. Jiménez, J.E. \& Ortiz, M.R. (2000). Conciencia fonológica y aprendizaje de la lectura: Teoría, evaluación e intervención. Aplicación en el aula. Madrid: Ed. Síntesis, Vallehermoso.

11. Godoy, D.A. (2004). Evoluçao das habilidades fonológicas e de leitura e escrita brasileiras alfabetizadas em dois métodos distintos. $2^{\circ}$ Congreso Hispano Portugués de Psicología, Sept. 22-25, Lisboa, Portugal.

12. Bravo, L. (2005) Lenguaje escrito y dislexia: enfoque cognitivo del retardo lector. Santiago: Ediciones Universidad Católica.

13. Maluf, M. \& Barrera, S. (2003). Consciencia Metalingüística e alfabetizaçao: um estudo con crianças da primeira série do ensino fundamental: Psi Reflex Crí.,16: 491-502.

14. Yakuba, P. (1999). Prueba destinada para evaluar habilidades metalingüísticas de tipo fonológicas (Magíster). Santiago: Facultad de Educación de la Pontificia Universidad Católica de Chile.

15. Jiménez, J.E. \& Ortiz, M.R. (2000). Conciencia metalingüística y adquisición lectora en lengua española. Span J Psychol, Vol.3, 37-46.

16. Coloma, C.J. \& Infante, M. (2004). El conocimiento del lenguaje escrito en niños que inician la etapa escolar. Paideia, Vol. 37, 51-61.

17. Bravo, L.; Villalón, M. \& Orellana, E. (2003). Predictividad del Rendimiento de Lectura: El Segundo Año Básico. Psykhe, Vol. 12, n² 2, 29-36.

18. Bravo, L.; Bermeosolo, J. \& Pinto, A. (1993). Diferencias neuropsicológicas en niños con retardo lector severo de distinto nivel socioeconómico. Revista Chilena de Psicología, Vol.13, $31-40$.

$\begin{array}{ll}\text { Recibido } & : \text { Abril } 2007 \\ \text { Aceptado } & : \text { Mayo } 2007\end{array}$

